REVISTA PERUANA DE INVESTIGACIÓN EDUCATIVA $2012, \mathrm{No} .4, \mathrm{pp} .43-76$

Las aulas hospitalarias desde las percepciones $\mathrm{y}$ vivencias de los niños y adolescentes hospitalizados

Giselle Silva Panez, Ph.D

Fundación Telefónica

Recibido 10.10.12

Revisado 10.10.12

Aprobado 15.10.12 


\section{Agradecimientos}

Giselle Silva Panez es Doctora en Psicopatología de Niños y Adolescentes por la Universidad de Zürich y Licenciada en Psicología Clínica por la Pontificia Universidad Católica del Perú. Se desempeña como investigadora y consultora en temas asociados a niños y familias en situación de vulnerabilidad. Actualmente, es Asesora del Programa Proniño de Fundación Telefónica y autora de varios libros y publicaciones.

El presente estudio es parte de una investigación que fue llevada a cabo durante el año 2011 en el marco del Programa Aulas Fundación Telefónica en Hospitales, llamada "Las vivencias de los niños hospitalizados desde sus dibujos y testimonios. Un estudio cualitativo con enfoque de resiliencia y derechos del niño hospitalizado". Ha sido apoyado por Fundación Telefónica en convenio con el Instituto de Estudios Peruanos, a los cuales la autora desea expresar su agradecimiento. Agradecemos, también, a Verousckha Uchofen, asistente del estudio, y a Andrea Tataje Márquez por su apoyo en el procesamiento de datos y la edición de este artículo. 


\title{
Las aulas hospitalarias desde las percepciones y vivencias de los niños y adolescentes hospitalizados
}

\begin{abstract}
Resumen
Este artículo presenta y discute los resultados de un estudio que tuvo como objetivo conocer las vivencias y percepciones de pacientes pediátricos y adolescentes en torno a la hospitalización y su participación en las aulas hospitalarias a las que estaban asistiendo. A partir de un enfoque cualitativo y una aproximación metodológica múltiple, se analizaron 330 dibujos realizados por los pacientes sobre los derechos del niño hospitalizado. También, se llevaron a cabo observaciones de las aulas hospitalarias y se condujeron entrevistas con niños, adolescentes, padres de familia, docentes hospitalarios y miembros del personal de salud en hospitales peruanos. Adicionalmente, se realizó observaciones etnográficas y sesiones de "juego médico" con dos grupos de niños. Los hallazgos evidenciaron la alta valoración que estos actores brindan a las aulas hospitalarias, que son identificadas como un servicio educativo que cumple diversas funciones, como brindar soporte emocional para la superación de la enfermedad, la ansiedad y el proceso de adaptación en el centro de salud; promover nuevos aprendizajes y contacto con técnicas de información y comunicación (TIC), así como prevenir el aislamiento social. Se observó que las aulas hospitalarias estimulan en los pacientes una actitud activa y autónoma frente a sí mismos, que es favorable para su bienestar.Ello sugiere que la acción educativa de estas, en diferentes planos, promueve la resiliencia de los pacientes.
\end{abstract}

Palabras clave: Derecho a la educación, necesidades educacionales, derechos del niño, hospital, sistema educativo 


\title{
Hospitalized children's and adolescent's perceptions and experiences of hospital classrooms
}

\begin{abstract}
This paper presents and discusses the results of a study, which main purpose was to describe the experiences and perceptions of pediatric and adolescent patients about hospitalization and their involvement in hospital classrooms. From a qualitative a multi-methodological approach, 330 drawings of hospitalized children were analized. Also, observation of hospital classrooms and interviews were carried out in peruvian hospitals with children, adolescents, parents, teachers and medical staff . Results show that all these actors attached high value to the hospital classrooms and perceived them as an educational service with several functions and benefits like emotional support to overcome the disease, the anxiety and hospital adjustment; encouragement to new learnings and contact with information and communications technology (ICT), as well as a mechanism to prevent social isolation. It was observed that the hospital classrooms encourage in patients an active and autonomous attitude toward themselves, favorable to their well-being. Results suggest that educational action that hospital classrooms display in various dimensions foster resiliency among patients.
\end{abstract}

Keywords: Right to education, educational needs, rights of the child, hospital, educational system 


\section{Introducción}

E n el Perú, la Educación Hospitalaria no forma parte aún de una política pública que satisfaga el derecho a la educación de los más de 156000 niños y adolescentes menores de 15 años que, anualmente, son hospitalizados en el país (INEI, 2010). Cabe anotar que ello que sí ocurre en Europa, Norteamérica y países latinoamericanos, como Argentina y Chile.

Los niños y adolescentes hospitalizados conforman una población altamente vulnerable. Durante su internamiento -que puede ser de corta o larga duración-, los menores experimentan, además de la dolencia física, una súbita ruptura con su vida cotidiana, separación del entorno familiar y social, así como la interrupción de su escolarización. Uno de los problemas derivados de esta situación es que sus oportunidades educativas se ven mermadas. Esto último acarrea, para un número indeterminado de niños y adolescentes, retraso o pérdida del año escolar, así como aislamiento social y la aparición de estados de ansiedad (Coyne, 2006; Ortiz, 2006).

Si bien la hospitalización es un tema altamente sensible por sus implicancias educativas, psicológicas y sociales en la vida de una gran cantidad de menores, este campo de estudio ha sido poco explorado desde la investigación académica. Tanto a nivel nacional como internacional, es muy limitada la literatura científica sobre intervenciones educacionales en el contexto hospitalario, la percepción que tienen los usuarios de estas y sus efectos sobre el curso educativo de los pacientes y su bienestar integral.

Es probable que la falta de atención brindada a la situación educativa de los niños y adolescentes hospitalizados en el Perú responda a que el tema no se haya problematizado en las agendas políticas de los sectores estatales competentes, pero también a que en los debates educativos no se le suela incluir y se priorice los temas vinculados a la educación básica regular. No obstante, desde el año 2000 existen en el Perú iniciativas privadas de pedagogía hospitalaria, como es el caso del Programa Aulas Fundación Telefónica en Hospitales (AFTH) y Aprendo Contigo (AC).

Este artículo presenta los resultados de un estudio de investigación de corte cualitativo que se realizó en el marco del programa AFTH. Este tuvo como uno de sus objetivos comprender la experiencia y las percepciones que los pacientes pediátricos y los miembros de la comunidad hospitalaria tienen sobre las aulas hospitalarias que funcionan en los centros de salud de los que forman parte.

La relevancia de este estudio está dada, principalmente, por la necesidad de alimentar un cuerpo de evidencias académicas que respalden la instauración de dispositivos de atención educativa en los hospitales como parte de 
una política pública integral e inclusiva. En ese sentido, la presente investigación ha centrado sus interrogantes en el valor, el significado subjetivo y las funciones que tienen las aulas hospitalarias para los alumnos-pacientes usuarios y los miembros de la comunidad hospitalaria.

Este trabajo tiene como base una versión más amplia del estudio. Este, además de lo concerniente a las aulas hospitalarias, exploró las vivencias de los pacientes con respecto a su situación de internamiento, así como los derechos y necesidades que priorizan acordes a su situación. Para efectos de elaborar el presente artículo, retornamos a las bases de datos originales para profundizar más en el tema que aquí nos ocupa. De este modo, es posible obtener nuevas evidencias que trascienden los hallazgos encontrados en la primera versión de la investigación.

\section{Marco teórico}

La mayoría de estudios científicos sobre la situación de los niños y adolescentes hospitalizados se ubican en revistas académicas de Enfermería Pediátrica, Pediatría y Psicología. Tratan temas como el estado emocional de los pacientes, la respuesta al dolor y el estrés, así como estrategias de afrontamiento (Board, 2005, Coyne, 2006). No obstante, los expertos en pedagogía hospitalaria vienen construyendo desde hace poco más de una década un cuerpo bibliográfico que integra el conocimiento proveniente de las intervenciones empíricas sostenidas, los estudios científicos y la reflexión académica (Lizasoáin, 2000; Ortigosa Quiles y Méndez Carillo, 2000; Guillén y Mejía, 2002).

En el ámbito nacional, la investigación en torno a la situación de hospitalización pediátrica es incipiente, pero revela la repercusión de variables emocionales en la recuperación física del paciente y la necesidad de prevenir la depresión infantil hospitalaria. En un estudio experimental con 156 niños y adolescentes peruanos, Drassinower (2006) halló que aquellos pacientes deprimidos poseían un tiempo mayor de hospitalización después de la operación en comparación con los no deprimidos, así como una mayor frecuencia de complicaciones. El estudio sugiere que factores como el dolor postoperatorio, los horarios de visita restringidos -contextualizados en una situación de cambio de las condiciones de vida producidos por una cirugía y asociados a una falta de soporte emocional- contribuyen a una mayor incidencia de trastornos depresivos, aunque estos sean reactivos.

En una exhaustiva investigación bibliográfica, Ortiz (2006) advirtió la existencia de tan solo 57 referencias útiles y relevantes relacionadas a te- 
mas psicológicos, educativos o sociales que se asocian con la hospitalización pediátrica.Ello fue posible luego de una depuración llevada a cabo sobre la base de 456 archivos encontrados entre los años 1948 y 2006 . El estudio de contenido de los artículos hallados develó el carácter teórico y empírico de los mismos. En este marco, se encontróla carencia de investigaciones que indagaran, por ejemplo, sobre el impacto y/o efecto de los programas educativos existentes en los centros de salud y las consiguientes técnicas de intervención sobre variables cognitivas, emocionales y familiares de pacientes pediátricos de corta estancia.

No obstante, Rappe, Abbott, y Lyneham (2006) demostraron en un estudio realizado a 267 pacientes pediátricos que un $15 \%$ más de estos redujeron su nivel de ansiedad luego de 12 a 24 semanas de biblioterapia para sus padres, donde se les ofrecía información y orientación pertinente sobre la situación de sus hijos. Por su lado, Serradas Fonseca, Ortiz González y Manueles Jiménez (2002) encontraron en Salamanca niveles más bajos de ansiedad en niños hospitalizados que asistían a aulas hospitalarias. Ello contrastaba con aquellos que no recibían atención educativa, lo cual sugiere la pertinencia de programas educativos para la población infantil hospitalizada.

Otros estudios disponibles han mostrado que son dos los tipos de factores del entorno hospitalario que influyen sobre el bienestar subjetivo de los pacientes pediátricos: la calidad del factor humano y el acceso a una infraestructura y recursos materiales que brinden oportunidad para el juego y el aprendizaje. González (2004) encontró que la alta valoración que los pacientes y sus padres otorgan a la experiencia de hospitalización se encuentra relacionada al carácter cercano, sensible y amable del trato que brinda el personal de salud, en contraposición a la desestimación de aquellas experiencias donde predomina la poca información, un inadecuado manejo del dolor, problemas de comunicación y el no disponer de espacios y oportunidades de juego (Velázquez, Caraballo, Picallos y Albar, 2009).

Por su parte, Board (2005) llevó a cabo un estudio sobre percepciones de los pacientes pediátricos durante su estadía en el hospital mediante dibujos y ha destacado la sensibilidad que los niños tienen frente a los comportamientos del personal de la salud y al desconocimiento de sus sentimientos.

Ello puede ser complementado con las indagaciones de Fernández y López (2006), quienes hallaron que el estrés infantil por hospitalización no se encuentra necesariamente relacionado con el tipo de valoración de las experiencias previas de internamiento, sino con las características actuales del entorno que rodea al paciente, sobre todo, con aquellas vinculadas al estado emocional de los padres del niño. 


\section{¿Qué es la Pedagogía Hospitalaria?}

La educación hospitalaria es una rama diferencial reciente de la Pedagogía, que se ocupa de la educación del niño enfermo y hospitalizado (Lizasoaín, 2000). Si bien, el primer proyecto piloto de escuela hospitalaria se dio en Viena en 1917, no es hasta la década los 80 que se empiezan a fundar las primeras asociaciones de pedagogos hospitalarios en Europa y, en 1986, el Parlamento Europeo presenta la Carta de los Derechos del Niños Hospitalizado. El primer libro en lengua castellana Pedagogía Hospitalaria. Actividad educativa en ambientes clínicos data de 1999 (González-Simancas y PolainoLorente, 1999).

Esta nueva rama, la educación hospitalaria, es concebida para algunos como parte de la educación especial. Desde esta perspectiva, las aulas hospitalarias se definen como establecimientos educacionales que buscan compensar la desigualdad educativa que se genera en torno a los niños enfermos, debido a los períodos de hospitalización a los que se enfrentan. Desde esta perspectiva, los objetivos de las aulas hospitalarias son garantizar la continuidad de los estudios, responder a las necesidades educativas de los alumnos y reincorporar al niño a su escuela de origen. De este modo, se evita la marginación y el asilamiento, sin perder de vista los aspectos cognitivos, emocionales y sociales que lo caracterizan y, en ocasiones, menoscaban su aprendizaje (Unesco, 2006).

Otras perspectivas complementarias la enfocan como una modalidad de la Pedagogía Social, que proporciona apoyo emocional al niño y a la familia, contribuye a prevenir los efectos negativos de la hospitalización, disminuye su ansiedad y procura mejorar la adaptación y el ajuste del paciente pediátrico (Polaino Lorente y Lizasoáin, 1992; Ortiz, 2006).Desde esta perspectiva, la Pedagogía Hospitalaria trasciende el currículo escolar, puesto que constituye un acompañamiento (Violant, Molina y Pastor, 2009). No obstante, cabe resaltar la anotación de Lieutenant (2006), quien ha señalado que la escuela o el aula hospitalaria no es terapéutica en sí misma, pero tiene efectos terapéuticos y que el profesor y el aula simbolizan la vida normal del paciente.

En términos generales, las aulas y escuelas hospitalarias se encuentran bajo un enfoque de educación inclusiva, pues lo que se busca es insertar al sistema educativo a los niños y adolescentes que se encuentran internados. De esta manera, se promueve en ellos una mejor calidad de vida; ello parte de la asunción de que todo niño tiene derecho a la educación, independientemente de su condición de salud. Desde esta premisa, Lizasoáin y Lieutenant (2002) postulan que un fin primordial de la Pedagogía Hospitalaria es que el 
niño hospitalizado pueda ser resiliente a la experiencia y que esta le sirva de aprendizaje para la vida al aprender a afrontar situaciones adversas de manera positiva.

A partir de ello, Lieutenant (2006) sostiene que el aula hospitalaria tiene una doble función: por un lado, promueve el mantenimiento del nivel escolar, la continuidad del aprendizaje y la reconciliación con la escuela; y, por el otro, tiene un valor terapéutico, puesto que -a través de su práctica- el docente hospitalario incentiva en el niño la actividad y la autonomía, el placer del conocimiento y el deseo de aprender y comunicarse. Al ayudarlo a proyectarse hacia el futuro, la escolarización es un factor de pronóstico positivo.

Finalmente, se debe recalcar la importancia de esta rama, lo cual parte de la premisa de que la educación es un derecho para todos los individuos. Los pacientes pediátricos son niños con necesidades educativas especiales que requieren de un modelo multidisciplinario de atención educativa flexible y la formación profesional específica del docente hospitalario.

\section{Resiliencia, vulnerabilidad y fortaleza del niño hospitalizado}

Si bien la enfermedad y la hospitalización es concebida como una experiencia perturbadora que sume al niño en una situación de crisis, desde el enfoque de la resiliencia, también es una oportunidad de aprender a superar con éxito situaciones adversas y poner en marcha recursos internos para el logro de una óptima adaptación en un entorno diferente.

Méndez Carillo y Ortigosa Quiles (2000) han señalado que los niños en situación de hospitalización suelen sufrir alteraciones psicológicas diversas, como desórdenes del sueño y de la alimentación, trastornos de conducta y pérdida de los niveles de aprendizaje y de los logros sociales previamente adquiridos. Sin embargo, también han llamado la atención sobre la variabilidad de reacciones de los niños ante dicha situación. Los autores advierten que el tipo de respuesta que el paciente desarrolla respecto a la hospitalización depende en gran medida de su temperamento y otros factores personales y ambientales. Dentro de este esquema, llaman factores amortiguadores de la experiencia hospitalaria a aquellos que promueven la adaptación exitosa, tales la autoestima, la capacidad para relacionarse, la iniciativa, el humor y la creatividad. Estos, aunados al juego y a situaciones de aprendizaje, favorecerían la resiliencia hospitalaria y un enfrentamiento exitoso de la situación.

En ese sentido, se podría afirmar que la capacidad resiliente en el hospital surge como resultado del interjuego de variables personales, sociales y ambientales, las que contrarrestan a aquellos factores de riesgo presentes. 
De ser el caso, el niño sería capaz de transformar la experiencia negativa en una de aprendizaje y fortalecimiento personal.

\section{Objetivo del estudio}

El objetivo de este estudio es describir las vivencias y las percepciones que los pacientes pediátricos y adolescentes, así como los miembros de la comunidad hospitalaria, tienen sobre las aulas hospitalarias que funcionan en los centros de salud de los que forman parte.

\section{Metodología}

Esta investigación de enfoque cualitativo utilizó una aproximación metodológica múltiple, que combina varias técnicas de recolección de datos. Estos últimos fueron triangulados (Docket y Perry, 2005).

La fuente principal de datos fueron los dibujos sobre los Derechos del Niño Hospitalizado, que fueron realizados por 330 pacientes pediátricos y adolescentes en el marco del concurso de pintura que organiza cada año el programa Aulas Fundación Telefónica en Hospitales para sus alumnos. Se tomó como unidad de análisis principal el dibujo que cada paciente realizó. Las producciones artísticas que se tomaron en cuenta fueron las realizadas en las versiones 2008,2009 y 2010 del mencionado concurso.

La participación de cada estudiante fue de carácter voluntario. Los participantes trabajaron previamente con sus docentes el tema de los derechos del niño hospitalizado, los conocieron y discutieron antes de realizar sus producciones. La consigna que los pacientes recibieron para realizar sus trabajos fue elegir el derecho que tuviera para sí mismo mayor significado, y que lo represente libremente. De esta forma, la primera estrategia para conocer las percepciones de los niños respecto a su experiencia de hospitalización y de participación en el aula fue el análisis psicográfico de los dibujos mencionados (Panez, 2004).

Como una estrategia complementaria al análisis de los dibujos, se realizaron en mayo de 2011 cuatro visitas de campo a hospitales en los cuales habían estado internados los participantes. Ello se ejecutó con el fin de llevar a cabo observaciones de las aulas, reconocimiento del ambiente hospitalario, entrevistas a algunos niños y adolescentes internados ${ }^{1}$, personal de salud

1. La mayoría de pacientes visitados eran niños que, recientemente, habían sido hospitalizados. Algunos, sobre todo los internados en el Hogar Clínica San Juan de Dios de Cusco, 
y padres de familia. Las visitas permitieron contextualizar los contenidos y mensajes de los dibujos analizados, así como profundizar en la comprensión de las vivencias que se iban identificando en el análisis de los mismos con respecto a la hospitalización y la participación de los pacientes en las aulas.

\section{Participantes}

\section{Análisis de los dibujos}

De los 330 pacientes que realizaron dibujos, 151 (46\%) fueron mujeres y 179 (54\%) eran varones. Los lugares de procedencia, las patologías y el tiempo de hospitalización fueron diversos. Al momento de realizar su producción gráfica, los participantes tenían entre tres y dieciocho años de edad, con una distribución que se aprecia en la siguiente tabla:

Tabla 1

Distribución de los participantes por edad

\begin{tabular}{lcc}
\hline \multicolumn{1}{c}{ Grupo de edad } & $\mathrm{n}$ & Porcentaje \\
\hline De 3 a 5 años & 21 & 7 \\
De 6 a 8 años & 77 & 23 \\
De 9 a 11 años & 85 & 26 \\
De 12 a 14 años & 79 & 24 \\
De 15 a 18 años & 60 & 18 \\
No especifica edad & 8 & 2 \\
\hline$N=330$ & &
\end{tabular}

Los participantes procedían de ocho hospitales estatales ubicados en seis provincias del Perú: Arequipa, Cusco, Chiclayo, Huancayo, Lima y Loreto. Los dibujos fueron realizados en las AFTH, ubicadas en diversos pabellones dentro de cada hospital. A continuación, presentamos cuál fue la distribución de los participantes por provincia y hospital. 
Tabla 2

Distribución de participantes por provincia y hospitales de procedencia

\begin{tabular}{lcc}
\hline Provincia & Hospital & \\
\hline Arequipa & Hogar Clínica San Juan de Dios & 40 \\
Chiclayo & Hogar Clínica San Juan de Dios & 10 \\
Cusco & Hogar Clínica San Juan de Dios & 36 \\
Lima & Hogar Clínica San Juan de Dios & 18 \\
lquitos & Hospital Regional de Loreto & 18 \\
Huancayo & Hospital Daniel Alcides Carrión & 19 \\
Huancayo & Hospital El Carmen & 19 \\
Lima & Instituto de Salud del Niño (Traumatología) & 55 \\
Lima & Instituto de Salud del Niño (Quemados) & 22 \\
Lima & Hospital María Auxiliadora & 12 \\
Lima & Instituto Nacional de Enfermedades Neoplásicas (Neutropenia) & 13 \\
Lima & Instituto Nacional de Enfermedades Neoplásicas (Pediatría) & 4 \\
Lima & Instituto Nacional de Enfermedades Neoplásicas (Adolescentes) & 28 \\
Lima & Sin identificación al momento de su análisis & 14 \\
Otros & & 330 \\
\hline Total & & \\
\hline
\end{tabular}

Es relevante mencionar que la realidad de cada hospital es diferente, lo que se evidencia en la diversificación de patologías que atienden. Por ejemplo, en el INEN (Instituto Nacional de Enfermedades Neoplásicas), se atienden enfermedades de tipo oncológicas. En el caso de los hospitales Hogar Clínica San Juan de Dios, se atienden patologías crónicas discapacitantes, como parálisis cerebral, que generalmente mantienen al niño hospitalizado por meses o años. En otros casos, como elHospital General de Loreto, María Auxiliadora, Hospital Alcides Carrión, Hospital Cayetano Heredia de Lima y Hospital el Carmen de Huancayo se presentan patologías agudas e infecciosas. En el Instituto de Salud del Niño, participaron niños con dolencias traumatológicas y niños quemados. El carácter de la atención que brindan los hospitales es, también, distinta en cuanto al tipo de cobertura que tienen: nacional, regional o distrital.

Dado que en el momento de la realización de los dibujos no se tenía la intención de que estos se emplearan con fines de investigación, el programa AFTH no registró datos adicionales a los presentados. Sin embargo, se conoce que la situación cultural, social y lingüística de los niños que asisten a las aulas es variada, aunque predomina la población que vive en pobreza y pobreza 
extrema. El lugar de procedencia, también, es diverso: algunos viven en la misma ciudad en la que se encuentra el hospital donde han sido internados, y otros vienen de distritos alejados de la ciudad. En aquellos hospitales ubicados en provincias, hay frecuentemente casos procedentes de áreas rurales, lugares marcados por la pobreza, la lejanía, el aislamiento. Ello constituye una dificultad para que la familia pueda mantener contacto regular con el niño hospitalizado.

El tiempo de internamiento de los pacientes participantes fue, también, variable: algunos de ellos permanecieron en el hospital pocos días por presentar dolencias agudas. Otros permanecieron meses por sufrir situaciones graves que requerían de un largo tratamiento. Algunos niños, los que provienen del Hogar Clínica San Juan de Dios de las diversas provincias, permanecieron o siguen aún internados, debido a que su dolencia es ya una discapacidad o la familia no puede hacerse cargo del tratamiento y recuperación del paciente.

\section{Visitas de campo}

Como se mencionó, cuatro hospitales fueron visitados con el fin conocer los entornos y aulas hospitalarias, entrevistar niños, adolescentes, padres de familia y personal de salud. Los hospitales visitados fueron: el INEN, el Hospital Cayetano Heredia en Lima, el Hospital Hogar San Juan de Dios del Cusco y el Hospital Regional de Loreto en Iquitos. Se eligió estos centros, debido a las diferentes realidades que representan. En total, se entrevistó a diecisiete pacientes, doce padres de familia, diez miembros del personal sanitario y cinco docentes hospitalarios.

A continuación, presentamos una tabla con el número de participantes en las visitas de campo. 
Tabla 3

Número de participantes en visitas de campo

\begin{tabular}{lccccc}
\hline Hospital & $\begin{array}{c}\text { Niño(as) y } \\
\text { adolescentes } \\
\text { entrevistados }\end{array}$ & Edad & $\begin{array}{c}\text { Padres/ } \\
\text { madres }\end{array}$ & $\begin{array}{c}\text { Personal } \\
\text { de salud }\end{array}$ & $\begin{array}{c}\text { Profesora } \\
\text { de Aula }\end{array}$ \\
\hline $\begin{array}{l}\text { Cayetano Heredia, } \\
\text { Lima }\end{array}$ & 8 & $6-11$ & 8 & $5^{*}$ & 1 \\
$\begin{array}{l}\text { Regional, Loreto } \\
\begin{array}{l}\text { San Juan de Dios, } \\
\text { Cusco }\end{array}\end{array}$ & 6 & & $3^{* *}$ & 2 \\
INEN, Lima & 3 & $16-17$ & & $2 * * *$ & 1 \\
\hline
\end{tabular}

* Un doctor, tres enfermeras y una técnica

**Una doctora, una enfermera, una psicóloga

*** Dos enfermeras

\section{Instrumentos y técnicas en las visitas de campo}

Para las visitas de campo a los hospitales, se emplearon técnicas provenientes de la psicología y la antropología, como la observación etnográfica de las aulas y las sesiones educativas, la visita de reconocimiento de los pabellones hospitalarios y los pacientes internados, las entrevistas individuales con adolescentes internados seleccionados, entrevistas grupales con padres de familia y personal de salud, y, finalmente, la sesión de juego diagnóstica aplicada a dos grupos de niños en la variante del juego médico (González, Benavidez y Montoya, 2000).

Se construyeron guías para la toma y recojo de información. También, se contó con un registro para anotar información y, en la mayoría de casos, se grabaron las entrevistas con el permiso de los participantes y se filmaron las sesiones de juego de los niños para poder analizarlas posteriormente con mayor fidelidad. En paralelo, se realizaron fotos de las sesiones de juego y de los niños (ello, con el cuido necesario para no distraerlos o interferir su actividad lúdica).

En el siguiente cuadro, mostramos las técnicas que aplicamos en cada hospital visitado: 
Tabla 4

Técnicas empleadas en el trabajo de campo

\begin{tabular}{|l|c|c|c|c|c|c|c|}
\hline $\begin{array}{l}\text { Técnica/ } \\
\text { Hospital }\end{array}$ & $\begin{array}{c}\text { Observación } \\
\text { de Aula } \\
\text { Telefónica }\end{array}$ & $\begin{array}{c}\text { Sesión } \\
\text { de juego } \\
\text { médico }\end{array}$ & $\begin{array}{c}\text { Entrevista } \\
\text { individual } \\
\text { a niños }\end{array}$ & $\begin{array}{c}\text { Entrevista } \\
\text { individual a } \\
\text { adolescentes }\end{array}$ & $\begin{array}{c}\text { Entrevista } \\
\text { grupal a } \\
\text { padres }\end{array}$ & $\begin{array}{c}\text { Entrevista } \\
\text { a } \\
\text { educador } \\
\text { de Aula }\end{array}$ & $\begin{array}{c}\text { Entrevista } \\
\text { a personal } \\
\text { de salud }\end{array}$ \\
\hline $\begin{array}{l}\text { Cayetano } \\
\text { Heredia, } \\
\text { Lima }\end{array}$ & $\checkmark$ & $\checkmark$ & $\checkmark$ & $\checkmark$ & $\checkmark$ \\
\hline $\begin{array}{l}\text { Regional, } \\
\text { Loreto }\end{array}$ & $\checkmark$ & $\checkmark$ & $\checkmark$ & & $\checkmark$ & $\checkmark$ & $\checkmark$ \\
\hline $\begin{array}{l}\text { San Juan } \\
\text { de Dios, } \\
\text { Cusco }\end{array}$ & $\checkmark$ & $\checkmark$ & $\checkmark$ & & & $\checkmark$ & $\checkmark$ \\
\hline $\begin{array}{l}\text { INEN, } \\
\text { Lima }\end{array}$ & $\checkmark$ & & & $\checkmark$ & & $\checkmark$ & $\checkmark$ \\
\hline
\end{tabular}

\section{Procesamiento de datos y análisis}

El procesamiento de los datos tuvo tres fases:

- Análisis de los dibujos

- Análisis de datos de las visitas de campo

- Integración de información en categorías temáticas

A continuación, se describen los pasos metodológicos de cada una de las fases:

\section{Análisis de los dibujos}

Como primer paso, se hizo una revisión inicial de los dibujos para observar representaciones recurrentes y temas prevalentes de los dibujos. Dada la diversidad de las características demográficas de los participantes, se optó por analizar aquellos aspectosen común y homogéneos en cuanto a los elementos que eligieron representar. Posteriormente, se identificaron indicadores gráficos y categorías de análisis para su sistematización cuantitativa. A continuación, se muestra un cuadro con las categorías cuantificables para el análisis de los dibujos: 
Tabla 5

Categorías de análisis cuantificable de los dibujos

Categoría

1. Derechos del niño hospitalizado más representados
Definición

El ranking determinado por la frecuencia de aparición de dibujos que representan uno de los 10 derechos del niño hospitalizado.

2. Vivencia representada en el di- El conjunto de características que expresan una bujo situación emocional determinada, ya sea positiva o negativa; alegría-disfrute, gratitud, desconfianza, tristeza, dolor, temor entre otros.

3. Tipo de relación con el adulto

El tipo de relación del personaje infantil representado con un adulto representado. Relación positiva o negativa expresada por el tipo de emoción que predomina entre ambos.

4. Adulto más representado

Ranking en términos de frecuencia de aparición de los personajes adultos representados por el niño o adolescente en el dibujo.

5. Rasgos de inseguridad, miedo, La presencia de piso detallado, inclinación de los angustia presentes en el dibujo personajes en más de 15 grados, repaso de la línea, líneas fragmentadas en los trazos de los dibujos (Koppitz, 1982)..

6. Escenario más representado

Ranking de frecuencia de aparición de los lugares representados por los niños o adolescentes en sus dibujos.

7. Elementos resilientes

Posición de parado, echado o sentado del personaje central del dibujo.

Representación de la enfermedad de forma manifiesta.

Presencia de sonrisa y actitud optimista en los personajes del dibujo.

\section{Análisis de datos de las visitas de campo}

En segundo lugar, se llevó a cabo la transcripción y análisis de las entrevistas realizadas a niños, adolescentes, padres de familia, docentes de aula y personal de salud. Se hizo lo propio con las sesiones de juego médico y se sistematizaron las notas de las observaciones de las aulas y entornos hospitalarios. 


\section{Integración de información en categorías temáticas}

En tercer lugar, se integró los datos de los momentos anteriores. A partir de una plataforma de sistematización cuantitativa y después de haber analizado cualitativamente los temas recurrentes de los dibujos y las visitas de campo, se creó un conjunto de categorías temáticas para integrar la información e interpretarla. Con el apoyo de estas categorías, se volvió a revisar por segunda vez los dibujos y se realizó una lectura cualitativa de los mismos. Las nuevas categorías permitieron un análisis cualitativo de los contenidos globales y mensajes escritos que aparecían en una gran cantidad de dibujos. De esta forma, se accedió a las vivencias predominantes y la calidad de las relaciones humanas representadas, sobre todo, con el personal de salud y los docentes de las aulas hospitalarias que fueron representados.

Seguidamente, se presenta un cuadro con las categorías cualitativas con las que se trabajó.

Tabla 6

Categorías de análisis cualitativo

1. Miedo, ansiedad e inseguridad en la situación de hospitalización

2. Necesidad de cariño, cuidado y atención

3. Resiliencia en el hospital: La fortaleza y el impulso hacia la vida, el juego y movimiento

4. La experiencia de los niños hospitalizados en las aulas hospitalarias

5. Derecho a que las propias necesidades (y el yo) sean reconocidos

6. Derecho al nombre: fundamento de la identidad

7. La relación del niño con su dolor

8. Derecho al acompañamiento de los padres durante la hospitalización

\section{Resultados}

\section{Los derechos del niño hospitalizado más representados}

En primer lugar, se presentan los resultados que dan cuenta de los derechos del niño hospitalizado; estos fueron elegidos con mayor frecuencia por los participantes como tema de dibujo. De los diez derechos, tres fueron los que ocuparon los primeros lugares, tal como se observa en la Tabla 7. 
Tabla 7

Derechos más representados en los dibujos

\begin{tabular}{clc} 
No de Derecho & \multicolumn{1}{c}{ Derecho más representado } & $\%$ \\
\hline 1 & $\begin{array}{l}\text { Que me llamen por mi nombre, me sonrían, me } \\
\text { acaricien, y me presten cuidado y atención } \\
\text { Que me permitan caminar, jugar, aprender y hacer } \\
\text { bullicio, si esto no interfiere con la calidad de mi } \\
\text { cuidado } \\
\text { Que pueda disponer de juguetes, libros, medios } \\
\text { audiovisuales adecuados a mi edad }\end{array}$ & 18 \\
\hline
\end{tabular}

$N=330$

La Tabla 7 muestra que el derecho a ser llamado por el propio nombre y ser atendido con cariño, cuidado y atención (Derecho 1) fue el más elegido; este concentra al $21,5 \%$ de participantes. El derecho de aprender, jugar, caminar y hacer bullicio (Derecho 5 ) y el de disponer de juguetes, libros y medios audiovisuales (Derecho 10) obtuvieron el segundo (18\%) y tercer lugar (15\%) respectivamente De ello, se deriva que una tercera parte (33\%) del total de participantes colocaron como prioridad sus necesidades educativas y lúdicas. Este resultado sugiere que estas necesidades son fundamentales para los niños y adolescentes en el marco hospitalario.Los otros siete derechos elegidos por el resto de participantes (45\%) hacen alusión a diversas necesidades, como ser reconocido y tratado como persona y no como caso; la presencia continua de los padres; la importancia de información clara y atención oportuna; la expresión libre y correspondida del dolor y la ansiedad, entre otros.

Dentro de este marco, no se encontraron diferencias de frecuencia por edad ni por sexo al momento de elegir el derecho a ser representado. En el análisis por centro hospitalario, tampoco se presentaron divergencias, a excepción de una tendencia mayor en los pacientes de la Clínica San Juan de Dios de las diversas provincias a privilegiar el derecho 5, asociado a la necesidad de juego y movimiento. Esto último sugiere que la cualidad de sus dolencias -que comprometen la movilidad del cuerpo- refleja la necesidad de actividad cinestésica y lúdica.

En la siguiente ilustración, se muestra el dibujo de uno de los participantes sobre la necesidad de juego. 
Ilustración 1

"iA su marca, listos, fuera!". "Qué divertido, te voy a alcanzar". "Ya voy a ganar".

"Los han operado, se han olvidado de su dolor". "Que me permitan jugar".

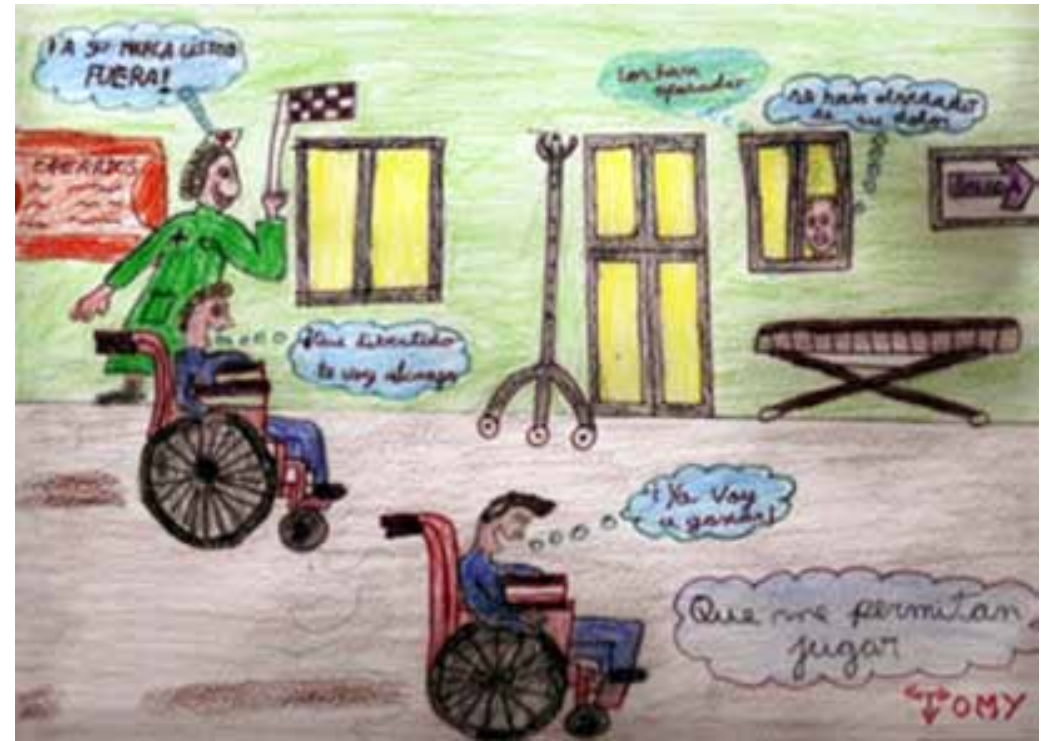

Alberto, 11 años

Alberto, de 11 años, señala en un breve párrafo que acompaña a su dibujo por qué decidió representar el derecho elegido y el significado de su dibujo: "Está bien, que nos permitan jugar si ya estamos un poco mejor, pero con cuidado de no lastimarnos. Yo me dibujé junto con mi amigo, porque ya pasó nuestra operación y cuando nos divertimos nos sentimos mucho mejor".

\section{Las aulas hospitalarias}

Del total de los 330 dibujos, el 12\% (40) de participantes representó como escenario y temática principal las aulas hospitalarias. De estos, las mujeres lo hicieron con relativa mayor frecuencia que los varones, aunque podemos observar que esta diferencia es mínima. 
Tabla 8

Participantes que graficaron el aula hospitalaria por género

\begin{tabular}{rcc}
\hline Sexo & $\mathrm{n}$ & $\%$ \\
\hline Mujeres & 22 & 55 \\
Hombres & 18 & 45 \\
\hline Total & 40 & $100 \%$
\end{tabular}

El análisis de contenido de los dibujos muestra que las aulas son percibidas como un espacio alternativo y diferente al resto del hospital. Ello muestra rasgos recurrentes como los siguientes:

Los niños y adolescentes se representan siempre (en el $100 \%$ de los casos) en una posición activa y autónoma: jugando, leyendo, usando la computadora o divirtiéndose o en interacción con pares y docentes. Ello se opone a otros escenarios graficados, en donde se representan en posición pasiva (en cama, por ejemplo) y en una relación de dependencia respecto a quienes los atienden.

Por su relevancia y recurrencia, la posición activa y autónoma del personaje protagónico en el dibujo fue seleccionado en este estudio como un indicador de resiliencia hospitalaria, es decir, como aquella capacidad del paciente pediátrico de poder enfrentar la situación adversa que atraviesa con recursos internos positivos. Este resultado sugiere que el aula hospitalaria conlleva en los niños y adolescentes internados la emergencia de sus fortalezas personales y agencia personal frente al entorno, a sí mismos y a su enfermedad, constituyéndose en ese sentido en un factor que promueve resiliencia.

En la siguiente ilustración, se puede observar el contraste entre un dibujo en el cual se representan a los pacientes en posición activa y otro en el que se representa al paciente en posición pasiva y dependiente. Nótese que los escenarios son diferentes. En el primer dibujo, los niños están en el aula hospitalaria; y, en el segundo caso, en el cuarto con una técnica. 
Ilustración 2

Posición activa y autónoma en el aula vs pasiva y dependiente en la habitación

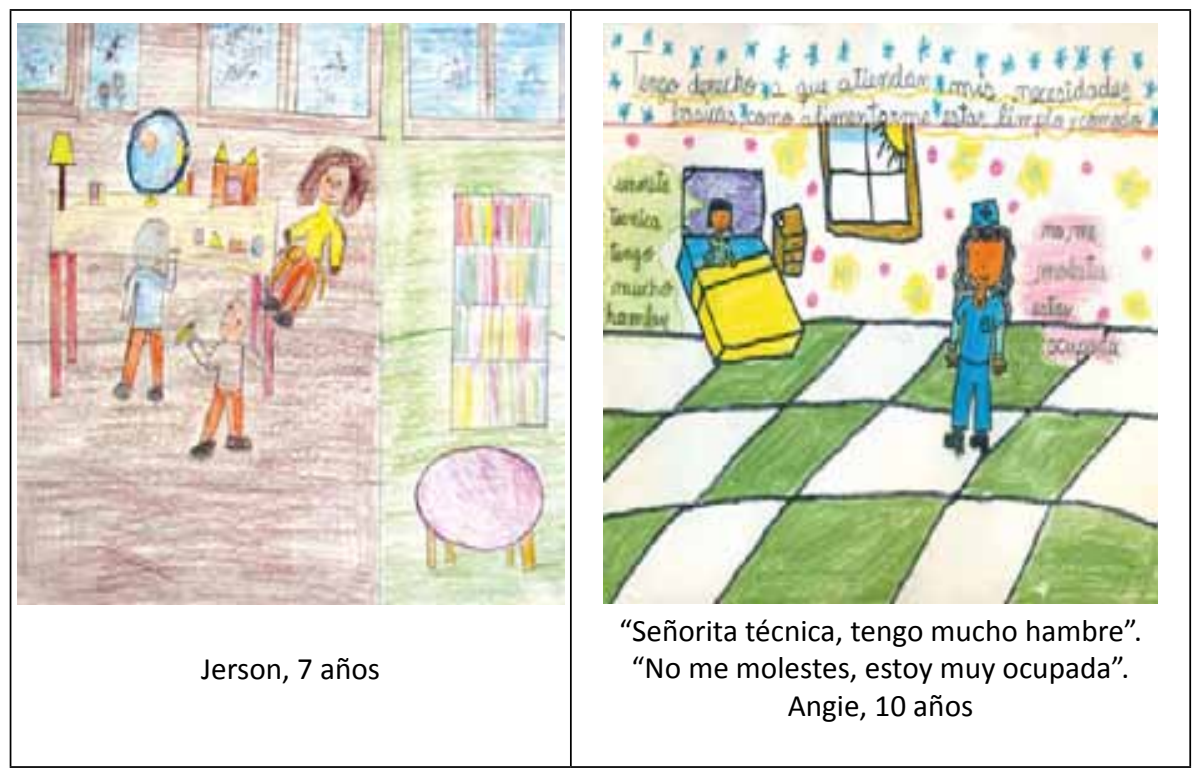

En el total de dibujos de aulas, hay una presencia significativa de materiales lúdicos y de aprendizaje como juguetes, libros, computadoras y televisor. Como se puede observar en la Tabla 9, en el $73 \%$ de dibujos de aulas, se representan computadoras o televisores. En el 53\%, aparecen libros y, en el $50 \%$, aparecen juguetes. Ello cual revela la importancia que le dan los pacientes a estos recursos educativos.

Tabla 9

Predominancia de tipo de material educativo representado ${ }^{2}$

\begin{tabular}{lcc} 
Tipo de material educativo & $\mathrm{n}$ & $\%$ \\
\hline Medios audiovisuales & 29 & 73 \\
Material de lectura & 21 & 53 \\
Material lúdico & 20 & 50 \\
\hline$n=40$ & &
\end{tabular}

Los recursos educativos y lúdicos, en especial las computadoras y los recursos audiovisuales, resultan ser altamente valorados por los pacientes, el

2. Dado que en los dibujos aparecen más de un tipo de material representado, la suma de los porcentajes supera el $100 \%$. 
personal de salud, los padres de familia y los docentes hospitalarios. En el siguiente dibujo, se puede apreciar el cuidado que el dibujante ha dedicado a representar todos los recursos educativos valorados.

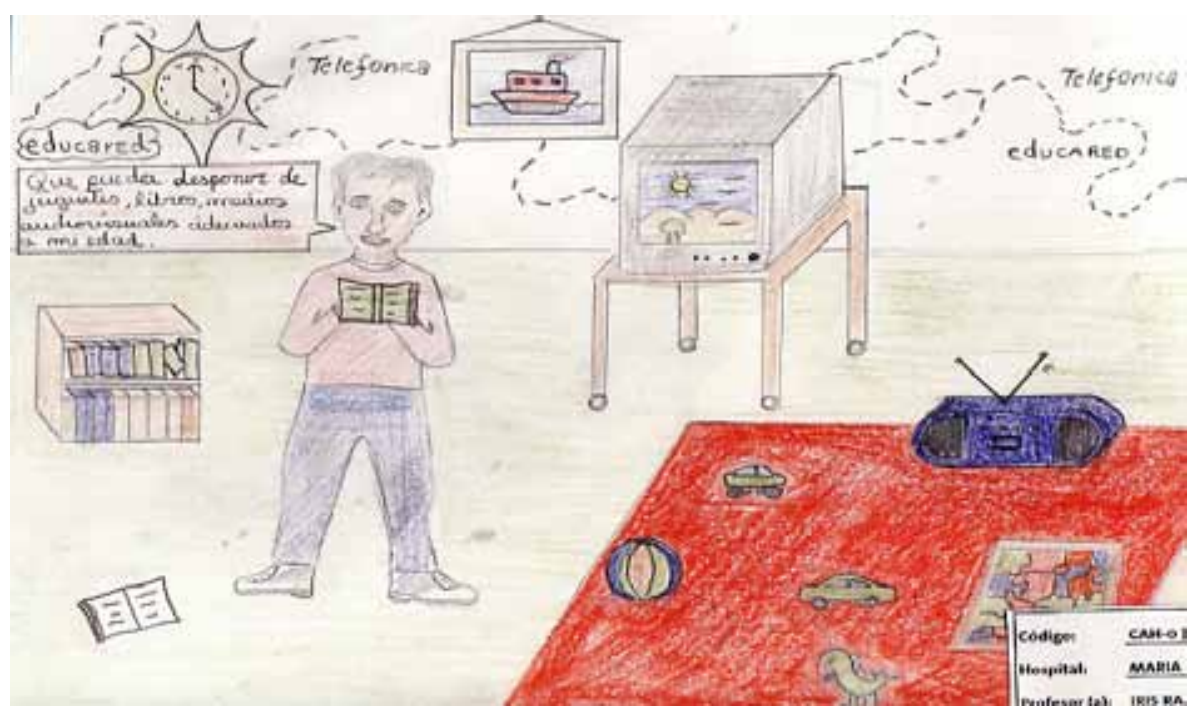

Los motivos por los cuales estos elementos son valorados se asocian a nuevas oportunidades de aprendizaje y al poder disponer de dispositivos novedosos respecto a los cuales el acceso es restringido por la situación de pobreza de la que proceden: "Ellos dibujan cosas como las manualidades, el Nintendo, el televisor, porque a veces no tienen eso en sus casas. La mayoría de ellos viene de provincia. Aquí descubren nuevas cosas, música, televisor y eso lo plasman en el dibujo"(Docente hospitalaria, INEN, Lima).

En cuanto a la situación de pobreza, es importante considerar el contraste que experimentan algunos pacientes entre las condiciones y comodidades del hospital y las de su casa, signadas por la carencia. El encuentro con nuevos idiomas y el lenguaje informático impactan a todos los niños, pero aún más a aquellos que, por su situación sociocultural, viven alejados del contacto con la lengua dominante y el acceso a la tecnología de las comunicaciones. De ello, dan cuenta algunas de las enfermeras: "Un niño nativo aprendió a dominar la computadora, incluso habló el castellano (...) ya no se quiso ir, lloró cuando se le dijo que estaba de alta (...)"(Enfermera, Hospital 
General de Loreto)."En el Aula los chicos tienen la posibilidad de aprender lo que es la computación, las redes y usarlas. Es importante el programa porque los niños aprenden más. Es una parte más de la educación de los chicos que fortalece su educación. Ellos además hacen lógico matemático y programas educativos"(Enfermera, San Juan de Dios, Cusco).

En los siguientes dibujos, se observa el valor que los recursos educativos tienen para los dibujantes. Estos son ubicados en espacios centrales y ocupan un área considerable del dibujo.

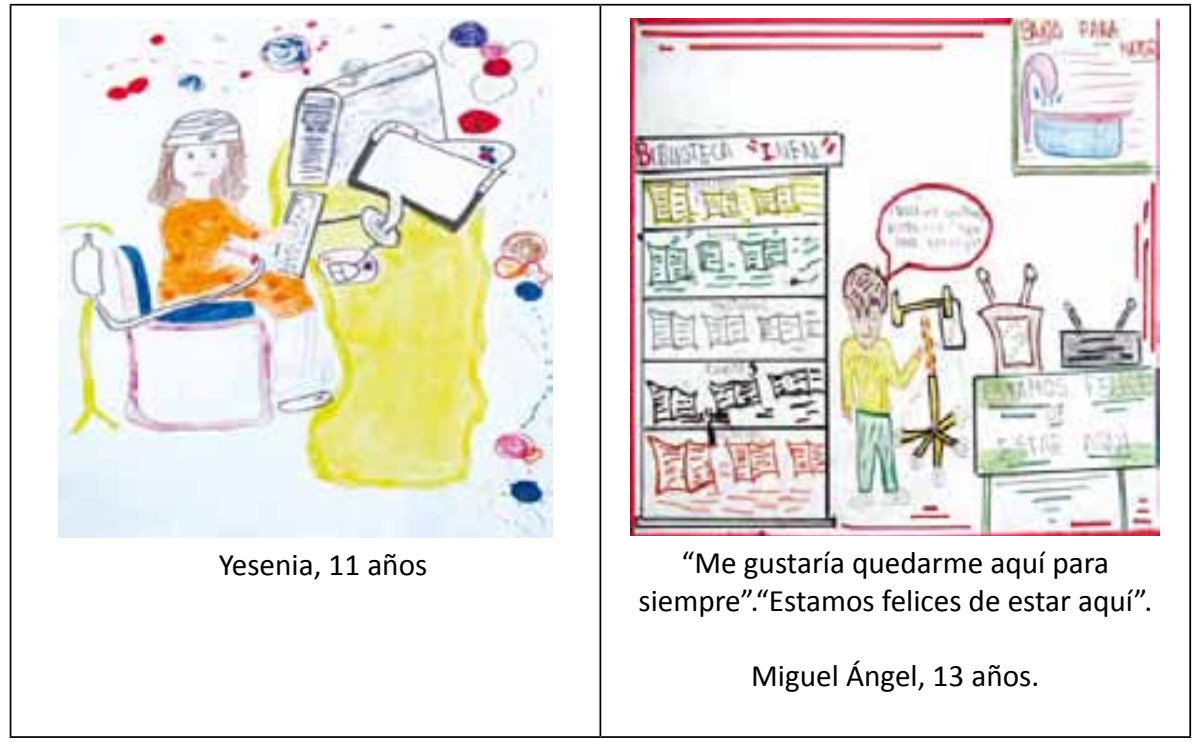

En los dibujos del aula, los pacientes se representan solos (50\%) o acompañados (50\%). La presencia de pares aparecen en el 30\% de los dibujos; el docente hospitalario, en el 25\%; y la enfermera, en el $15 \%$ de los dibujos.

En las ilustraciones en las que no se representa al aula, pero se hace referencia al juego y los recursos educativos, los personajes que acompañan al niño son médicos, enfermeras, técnicas, padres de familia y pares. Estos, también, son instados a jugar y participar de actividades educativas y lúdicas.

\section{Funciones y beneficios del aula hospitalaria}

Las funciones y beneficios del aula hospitalaria fueron mencionados recurrentemente por los diferentes participantes en el estudio. En este esquema, 
cada actor ha colocado un énfasis particular en uno u otro aspecto.Las funciones y beneficios manifestados por los diversos actores se sintetizan en la Tabla 10.

Tabla 10

Funciones y beneficios del aula hospitalaria según la percepción de los miembros de la comunidad hospitalaria

\begin{tabular}{|c|c|c|}
\hline Dimensión & Función & Beneficios para el paciente \\
\hline $\begin{array}{l}\text { Salud y bienestar físico } \\
\text { y mental }\end{array}$ & $\begin{array}{l}\text { Apoyo emocional } \\
\text { Normaliza la vida del paciente }\end{array}$ & $\begin{array}{l}\text { Genera bienestar } \\
\text { Reduce la ansiedad } \\
\text { Favorece la adaptación al } \\
\text { hospital } \\
\text { Favorece la recuperación física }\end{array}$ \\
\hline Educación & $\begin{array}{l}\text { Compensación de la } \\
\text { escolaridad interrumpida } \\
\text { Acceso a las TIC } \\
\text { Mediación de nuevos } \\
\text { aprendizajes } \\
\text { Acceso a recursos educativos } \\
\text { y lúdicos }\end{array}$ & $\begin{array}{l}\text { Se evita el retraso o la deserción } \\
\text { escolar } \\
\text { Aprendizaje de lengua castellana } \\
\text { y TIC }\end{array}$ \\
\hline Socialización & $\begin{array}{l}\text { Promueve la comunicación } \\
\text { con pares y adultos } \\
\text { Apoya la recreación } \\
\text { y el entretenimiento }\end{array}$ & Previene el aislamiento social \\
\hline
\end{tabular}

\section{La perspectiva de los pacientes}

El análisis cualitativo de la perspectiva de los niños y adolescentes sobre las funciones y beneficios de las aulas hospitalarias hacen alusión a tres aspectos: el bienestar emocional, la dimensión educativa y la esfera social. En las entrevistas, pudo observarse que el aula hospitalaria es para los pacientes un ambiente acogedor que trae beneficios emocionales y la posibilidad de establecer relaciones humanas afectuosas y gratificantes. Asimismo, los pacientes colocaron su atención en los aspectos educativos y las oportunidades de aprendizaje, así como en el acceso a recursos educativos estimulantes. Ello se puede corroborar a partir de las siguientes menciones: "El Aula me hace sentir que estoy bien, es como estar en casa. Es como una familia, nos hablamos"(Julia, 17, INEN). "Me gusta el aula, las computadoras, armar rompecabezas, dibujar, leer. Me gusta todo”(José, Hospital regional de Loreto).“Lo que más me gusta del hospital es el aula (...) Me gusta el aula, porque no me 
aburro, me divierte, todo me gusta. Te explican de la comunicación, trabajo de arte. Mi profesora es cariñosa, es muy alegre. Yo lo haría más grande, más computadoras e Internet"(Walter, Hospital Regional de Loreto).

\section{La perspectiva de los padres de familia}

En los testimonios de los padres de familia, se observó un énfasis en las tres dimensiones y se destacaron las aulas hospitalarias como un importante paliativo de la ansiedad del niño, pero también de la propia: "Es algo bonito, allí se entretienen, se olvidan un poco de su enfermedad. Se les ve más tranquilos, salen alegres. Nos tranquiliza también a nosotros"(Padres del Hospital Regional de Loreto).

También, dieron importancia al aula hospitalaria como instancia compensatoria de la escolaridad interrumpida. Los entrevistados expresaron el deseo de una enseñanza "como en el colegio", que cubra contenidos del currículo escolar para que "no pierdan clases y no se atrasen". Los padres resaltan: "Lo que sería bueno es también que aprendan matemáticas, que les hagan sus clases; a veces, les hacen.Eso es bueno para que no se atrasen" (Madre de familia del Hospital Cayetano Heredia).

Asimismo, se destaca el contacto y aprendizaje del manejo de las TIC, que apareció como un elemento valorado:"A él le gusta la computadora, ha aprendido a manejarla muy bien, antes no sabía usarla, aquí en el hospital ha aprendido. Eso es bueno; la informática es necesaria"(Padre de familia de Hospital Regional de Loreto).

\section{La perspectiva del personal de salud}

El personal de salud puso énfasis en los beneficios que las aulas traen para la salud y bienestar físico y mental de los pacientes, así como para la prevención del aislamiento social de los niños como consecuencia de la hospitalización. Además, identifican una función educativa portadora de efectos sociales en la vida de los pacientes y en la normalización de la misma. De igual modo, reportaron que las actividades pedagógicas contribuyen a fortalecer su autoestima:

Las aulas cumplen una función de educación. Cuando un niño se enferma, rompe su rutina y estar encerrado, es frustrante: no juegan [...]. El aula ayuda a que el niño no rompa su rutina y no se sienta frustrado por su falta de movimiento físico. En el aula, hacen actividades pedagógicas, ven películas, se comunican, se integran, hacen amigos, interactúan entre ellos, juegan, hay cuentos. Todo eso hace que puedan desarrollar sus activida- 
des normales. Y los que no pueden venir acá, la profesora está muy pendiente de ellos y lleva los dibujos a la cama. Si tú puedes observar, si vieras en las paredes... lo ponen sus dibujos en su cuarto, son como trofeos, un orgullo para ellos, y lo enseñan [...]. Esto alivia nuestro trabajo.(Enfermera del Hospital General de Loreto)

Por otro lado, el personal de salud es sensible y advierte que las aulas hospitalarias mejoran el estado anímico de los niños; de esta forma, promueven la recuperación de la salud. Los profesionales entrevistados identifican y reconocen las necesidades socioemocionales de los pacientes en el contexto hospitalario. Independientemente de que puedan ser atendidas en todos los casos, no son ajenos a esta dimensión.El aporte de las aulas, en ese sentido, termina por aliviarlos en el manejo de estos aspectos. Ello se manifiesta en las siguientes menciones:"El aula los hace más despiertos [...], se le acompaña, el cariño de las personas y la tecnología, las computadoras y los juegos. Con cariño evolucionan mejor"(Enfermera del Hospital Regional de Loreto). Un médico resalta: "El aula cumple este trabajo emocional importante, es un apoyo complementario [...].El niño no solo necesita el tratamiento médico, sino el cariño y el afecto [...]. La parte afectiva es lo primero" (Pediatra del Hospital Regional de Loreto). Finalmente, se expresa que "Las aulas son una distracción para los chicos. Ayuda a distraerse, saca del estrés, se olvidan de su suero, se despejan"(Enfermera del INEN).

En las entrevistas al personal de salud, también, se evidenció que perciben que las aulas favorecen la comunicación de los niños con sus pares y familiares. De este modo, se evita el aislamiento social causado por su condición.

\section{La perspectiva del docente hospitalario}

Para el docente hospitalario, el aula cumple funciones que trascienden lo estrictamente pedagógico. Las más resaltantes son que se facilita el proceso de adaptación hospitalaria y, complementariamente, apoya el restablecimiento físico de los alumnos-pacientes al brindar soporte a la dimensión socio emocional de estos. A su vez, aporta en gran medida a las necesidades de comunicación e integración social de los niños:

Para mí, el aula tiene tres funciones: una que es para comunicarse con la familia, los amigos. Ellos se comunican por el chat, la cámara. Otra, para distraerse, por ejemplo, cuando le ponen la vía; y la tercera, para informarse de temas que le agradan, informarse de su enfermedad, de su tratamiento. También, ellos vienen a conversar. Ellos dan soporte a los otros 
que vienen, les dicen qué les van a poner, qué van a sentir. Conversamos sobre todo esto. Ellos me cuentan todo: cómo ha sido su vida en provincia, qué extrañan del lugar de donde vienen. (Docente hospitalaria, INEN)

\section{El rol del docente hospitalario}

El docente hospitalario tiene un significado especial para el niño y su presencia es bienvenida. En los 10 dibujos en los que el docente ha sido representado (25\% de los dibujos de aulas), este aparece como un personaje afectuoso, al que se le asocia con el juego, y que es portador de recursos materiales lúdicos y educativos que simbolizan la vida normal del niño. Además, en las visitas a los hospitales, se observó que los niños suelen esperar con ansias el momento en el que llega el docente y se abre el aula hospitalaria:"Los niños están pendientes a qué hora viene la profesora. El aula fortalece al niño en su educación y su enfermedad. Mejora al niño"(Enfermera, San Juan de Dios, Cusco).

En el siguiente dibujo, se observa la alegría de los niños representados frente a la docente hospitalaria que trae materiales educativos.

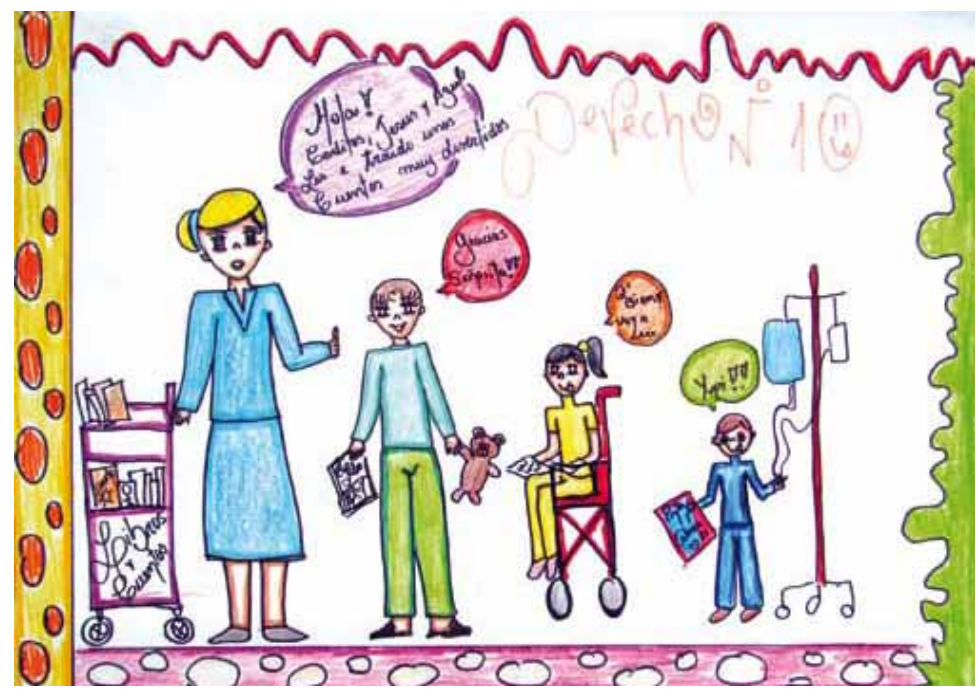


Por otro lado, los niños hacen constante referencia al buen trato que el docente les brinda, así como a la alegría y cariño que perciben por parte de estos: "Mi profesora es muy buena" (Carla, 8 años, Hospital Regional de Loreto), "Mi profesora es cariñosa, es muy alegre" (Jesús, 11 años, Hospital Regional de Loreto).

Respecto a ello, cabe anotar que la labor del docente dentro del aula hospitalaria va más allá del rol tradicional que se le adjudica en la escuela regular. Se convierte así en un apoyo emocional constante y fuente de motivación para los niños. "[...] El aula eleva la autoestima de los pacientitos, les hago que ver que es una persona importante para su familia y para su sociedad"(Docente hospitalario, Clínica San Juan de Dios de Cusco).

Los docentes hospitalarios cumplen, también, una función como promotores y tutores de resiliencia (Cyrulnik, 2004) al alentar a los pacientes a sobreponerse a la adversidad y ayudarlos a encontrar un sentido a su dolencia y proceso de recuperación. Ello se refleja en la siguiente mención:

Nosotros les enseñamos a los chicos que todo se puede en la vida, que podemos salir adelante a pesar de la adversidad; les damos las fuerzas. Todo eso hace que ellos tengan motivos en la vida, les decimos que ellos no son los únicos en la vida que están así, les contamos casos. Los chicos con discapacidad no deben quedar ahí; pueden hacer mucho más. Y se los decimos a ellos. Muchos de ellos se deprimen y nosotros les damos un aliento un apoyo.(Docente Hospitalario, Hogar Clínica San Juan de Dios de Cusco).

Tanto el aula hospitalaria como el docente se han convertido en una necesidad para el niño hospitalizado. En este espacio, cumplen el rol de intermediario entre el niño y los demás profesionales de la salud, lo que conlleva a una mejora de los canales de comunicación, así como a una óptima adaptación del niño al centro hospitalario. De ello, da cuenta el personal de salud cuando señala: "[...] ]ella (refiriéndose a la profesora) es un apoyo para nosotros porque nos ayuda en el tratamiento (....) lo que ella hace con los niños es complemento del trabajo"(Pediatra, Hospital General de Loreto). Respecto a ello, uno de los maestros señala: "La adaptación es por el apoyo que damos nosotros, el apoyo afectivo que les brindamos. Ese apoyo afectivo es lo que les permite que ellos salgan de sus ratos tristes pensando en sus padres, cambian todo eso" (Docente hospitalario, Hogar Clínica San Juan de Dios de Cusco). 


\section{Discusión e implicancias de política}

Este artículo presenta los resultados de una investigación académica sobre un tema que aún no se ha incorporado a las políticas públicas peruanas, como es la educación hospitalaria.

Basados en el análisis de 330 dibujos de pacientes pediátricos y adolescentes, entrevistas, observaciones y sesiones de juego de representación en hospitales, los hallazgos evidencian que las aulas hospitalarias son altamente valoradas por los pacientes y los miembros de la comunidad hospitalaria, y que promueven la resiliencia de los niños y adolescentes internados.

El estudio muestra que los beneficios percibidos por los usuarios directos e indirectos trascienden lo estrictamente pedagógico. Si perder su rol educativo, el aula parece tener efectos favorables en el proceso de adaptación hospitalaria, la disminución de la ansiedad y el restablecimiento de los pacientes, como resultado del soporte socioemocional que brindan.Gracias a ello, padres de familia y personal de salud, también, ven aliviada la propia tensión asociada. Los beneficios reportados se sitúan del mismo modo en la adquisición de nuevos aprendizajes, integración social y acceso a las TIC, y otros recursos educativos y lúdicos.

El estudio también llama la atención sobre capacidad resiliente de los niños y adolescentes, quienes aún en una situación adversa como es la hospitalización tienden naturalmente a orientarse hacia la acción, la participación y la autonomía, lo cual tiene implicancias de primer orden para el diseño de modelos educativos alternativos.

Esta investigación ha puesto de manifiesto que los beneficios reportados se sustentan en la alta prioridad que los pacientes le otorgan al aprendizaje, el juego, el movimiento y la calidad positiva de las relaciones humanas como pilares de su bienestar en el marco de la hospitalización.

Respecto a los factores valorados por los pacientes y sus padres en el hospital,los resultados concuerdan con lo hallado por González (2004), Board (2005) y Velázquez, Caraballo, Picallos y Albar (2009): estos son la calidad del trato del personal de salud y el acceso a recursos materiales que brindan oportunidad para el juego y el aprendizaje. Sin embargo, el estudio añade a lo anterior el papel que juega la educación hospitalaria en el mejoramiento de la calidad de vida de los pacientes y la valoración que tiene para estos la oferta educativa que ofrecen.

Si bien un aula hospitalaria propone actividades de aprendizaje, juego y movimiento en el marco de relaciones afectuosas y esto de por sí satisface necesidades y derechos intrínsecos de los pacientes, esto no debería limitarse solamente a su existencia. Tampoco, debería esperarse que solo a partir 
de un programa educativo los niños y adolescentes puedan gozar de estos derechos durante su internamiento.

Asimismo, los hallazgos del estudio demuestran que las necesidades de aprender, jugar y moverse son situadas más allá del programa educativo y los docentes hospitalarios. Los pacientes plantean en sus dibujos y testimonios que esperan que también médicos, enfermeras y técnicas se relacionen con ellos con afecto, actitudes pedagógicas y estrategias lúdicas. Del mismo modo, requieren durante el internamiento la provisión de material de lectura, de juego y oportunidades de movimiento, juego y aprendizaje.

Ello confronta a los centros hospitalarios pediátricos de nuestro sistema de salud con la necesidad de cuestionarse ciertos aspectos de su administración y gestión. Estos giran en torno a: (i) si los protocolos de atención y la organización de los horarios por los que se rige favorecen que los pacientes pediátricos satisfacen las necesidades mencionadas; (ii) si las actitudes y conocimientos del personal de salud alientan estas prácticas; (iii) si las áreas físicas y la organización del mobiliario y equipamiento se adaptan a que los niños puedan moverse, jugar y aprender de manera activa y autónoma.Sería necesario realizar estudios en esta dirección.

En realidad, nuestro sistema hospitalario, como el de muchos países, dista de contemplar la incorporación de aspectos que no sean de naturaleza estrictamente médica en sus protocolos de atención. A su vez, los hospitales no han considerado en sus diseños arquitectónicos y administrativos espacios para el aprendizaje y el juego de los pacientes pediátricos, como si lo educativo y lo sanitario no tuvieran puntos de encuentro.

Los resultados de este estudio sugieren la necesidad de poner en la agenda política el tema de la educación hospitalaria y que el Estado asuma su incorporación al sistema educativo oficial en el marco de una política educativa inclusiva. De esta forma, se garantizaría el derecho a la educación de estudiantes, quienes por su situación de salud se ven imposibilitados de asistir con regularidad a una institución educativa.

La pedagogía hospitalaria debe ser reconocida como una modalidad educativa articulada con las distintas áreas de educación inicial, primaria y secundaria del sistema educativo nacional. Si bien realizan un trabajo valorado y relevante para el bienestar de los pacientes y la comunidad hospitalaria, no es suficiente el alcance y la cobertura que tienen los proyectos que auspician las iniciativas privadas de aulas hospitalarias en el país. A pesar de contar con valiosos modelos pedagógicos sobre los cuales debería basarse la propuesta estatal, estos solo logran atender a un pequeño porcentaje de los más de 156000 pacientes menores de 15 años que son hospitalizados anualmente en el Perú (INEI, 2010). 
Debido a esto, es patente la necesidad de articular la educación hospitalaria al sistema educativo y convertirla en política pública. Sin embargo, esto no resultaría viable si no se desarrolla una visión articulada respecto a la atención integral que demanda la niñez en nuestro país en cuanto a sus necesidades educativas y sanitarias. Para ello, se necesita más investigación, particularmente, sobre las tasas de retraso, deserción y pérdida del año escolar en niños y adolescentes hospitalizados con el fin de conocer la magnitud del problema y poder planificar soluciones adecuadas. No obstante, la situación de la niñez hospitalizada supone un enfoque intersectorialobligatorio, así como la comprensión de que el derecho a la educación va mucho más allá de la escolarización. Este requiere un afronte flexible y una mirada sincera a la integralidad del niño.

Es necesario, luego,poder contar en las facultades de educación con la especialidad de pedagogía hospitalaria, que forme docentes para el trabajo con niños hospitalizados. Es un campo de trabajo muy fértil que demanda, sobretodo, vocación, pero además una solvente formación personal y académica. Por su parte, la formación y capacitación de los profesionales de salud debe incluir contenidos y metodologías vinculados a las necesidades emocionales y cognitivas de los pacientes pediátricos y adolescentes. El objetivo de ello no es suplir el papel del docente hospitalario, sino lograr con este un abordaje integral de la problemática del niño hospitalizado.

Se hace, también, necesario considerar los determinantes sociales de la salud y la enfermedad como eje del abordaje que se brinda a los problemas de la niñez y adolescencia peruana.Las visitas a los hospitales permitieron evidenciar variables sociales y culturales que determinan para muchos niños las causas de su enfermedad, su adaptación al hospital, las consecuencias negativas y positivas del internamiento y cómo interactúa la realidad del entorno del cual provienen con el curso de su situación posterior.

Como señala la OMS, la mayor parte de los problemas de salud se pueden atribuir a las condiciones socioeconómicas de las personas. Sin embargo, en las políticas de salud, han predominado las soluciones centradas en el tratamiento de las enfermedades, sin incorporar adecuadamente intervenciones sobre las "causas de las causas", tales como, por ejemplo, las acciones sobre el entorno social. En consecuencia, los problemas sanitarios se han mantenido, las inequidades en salud y atención sanitaria han aumentado y los resultados obtenidos con intervenciones en salud centradas en lo curativo siguen siendo insuficientes.

Una reflexión análoga tendría que hacerse respecto al problema educativo de la niñez peruana. Los determinantes sociales de los bajos niveles educativos no son suficientemente considerados en el abordaje y el diseño de políticas que atiendan a la gran diversidad de grupos estudiantiles peruanos, dentro de los cuales sen encuentran los alumnos hospitalizados. 


\section{Referencias}

Board,R.(2005). School-age children's perceptions of their PICU hospitalization.Pediatric nursing, 31(3), 166-175.

Cyrulnik, B. (2004). El realismo de la esperanza. Barcelona: Gedisa.

Coyne,I. (2006). Children's experiences of hospitalization.Journal of Child Health Care, 10(4), 326 - 336.

Drassinower, D. (2006). Depresión como factor pronóstico de la evolución post - quirúrgica en niños(Tesis de Medicina).Universidad Cayetano Heredia, Perú.

Docket, S., y Perry, B. (2005). Researching with Children: Insights from the Starting School Research Project.Early Development and Care,175(6).

Fernández, A. y López, I. (2006). Transmisión de Emociones, miedo y estrés infantil por hospitalización. International Journal of Clinical and Health Psychology, 6(3), $631-645$.

Gonzáles, P. (2005). Experiencias y necesidades percibidas por los niños y adolescentes con cáncer y por sus familias. Nure Investigación,16, 1-15. Recuperado de http://www.nureinvestigacion.org/FICHEROS_ADMINISTRADOR/ORIGINAL/Original\%2016.pdf

Gonzáles-Simancas, J. y Polaino-Lorente, J. (Comp.) (1990). Pedagogía Hospitalaria. Actividad educativa en ambientes clínicos. Madrid: Narcea.

González, R.; Benavides, G. y Montoya, I. (2000). Preparación psicológica basada en el juego. En: F. Ortigosa Quiles, J. yJ. Méndez Carillo (Coords.). Hospitalización Infantil. Repercusiones psicológicas. Madrid: Biblioteca Nueva.

Guillen, M. y Mejía, A. (2002), Actuaciones educativas en Aulas Hospitalarias. Atención escolar a niños enfermos. Madrid: Narcea Ediciones.

Instituto Nacional de Estadística e Informática (2010). Compendio estadístico. Información socio-demográfica/6. Salud E. Atenciones en salud. Recuperado de http://inei.gob.pe/Sis/index.asp

Koppitz, E. (1982).El dibujo de la figura humana en los niños. Evaluación psicológica. Buenos Aires: Guadalupe.

Lieutenant, Ch. (2006). La evolución de las escuelas hospitalarias: un camino por recorrer. Aulas Hospitalarias. Reflexiones de la VIII Jornada sobre pedagogía Hospitalaria (41 - 56). Recuperado de http://unesdoc.unesco. org/images/0015/001532/153274s.pdf

Lizasoaín, R. (2000). Educando al niño enfermo. Perspectivas de la pedagogía hospitalaria.Ciudad: Eunate. 
Lizasoáin, O. y Lieutenant, C. (2002). La Pedagogía Hospitalaria frente a un niño con pronóstico fatal: Reflexiones en torno a la necesidad de una formación profesional específica. Estudios sobre Educación (2), 157-165. Recuperado de, http://dspace.unav.es/dspace/bitstream/10171/8075/1/ Notas\%201.pdf

López, I., y Fernández, A. (2006, setiembre - diciembre). Hospitalización infantil y atención psico-educativa en contextos excepcionales de aprendizaje. Revista de Educación, 553-577.

Méndez Carillo, J. y Ortigosa Quiles, F. (2000). Estrés por hospitalización. En: J. Ortigosa Quiles, J. yF. Méndez Carillo. Hospitalización Infantil.Repercusiones psicológicas. Teoría y práctica (31-50). Madrid: Biblioteca Nueva.

Ortigosa Quiles, F. y Méndez Carrillo, J. (Coords.) (2000). Hospitalización Infantil. Repercusiones psicológicas. Teoría y práctica. Madrid: Biblioteca Nueva.

Ortiz, A. (2006, julio - diciembre). Ansiedad y miedos en niños ante la hospitalización. Investigación, intervención, programas y técnicas. Pensando Psicología. Revista de la Facultad de Psicología Universidad Cooperativa de Colombia, 3(3), 84- 100. Recuperado de http://wb.ucc.edu.co/pensandopsicologia/files/2010/09/articulo-11-vol3-n3.pdf

Panez, R. (2004). El lenguaje silencioso de los niños. Un estudio peruano sobre los derechos del niño desde su producción creativa. Lima: Panez y Silva Ediciones.

Polaino Lorente, A. y Lizasoáin, O. (1992). La pedagogía hospitalaria en Europa: La historia reciente de un movimiento pedagógico innovador. Psicothema, 4(1), 49 - 67. Recuperado de http://www.psicothema.com/ pdf/814.pdf

Rappe, R., Abbott, M. y Lyneham, H. (2006, junio). Bibliotherapy for Children With Anxiety Disorders Using Written Materials for Parents: A Randomized Controlled Trial. Journal of Consulting and Clinical Psychology, 74(3), 436 - 444.

Serradas, M., Ortiz, M. y De Manueles, J. (2002).Necesidades de asistencia educativa al niño hospitalizado. Enseñanza, 20, 243 - 258. Recuperado de http://e-spacio.uned.es/fez/eserv.php?pid=bibliuned:20382\&dsID=n ecesidad_asistencia.pdf

Unesco (2006). Aulas Hospitalarias. Reflexiones de la VIII Jornada sobre pedagogía Hospitalaria. Recuperado de http://unesdoc.unesco.org/ images/0015/001532/153274s.pdf 
Velázquez, R., Caraballo, J., Picallos, M. y Albar, M. (2009, octubre - diciembre). Experiencias de niños hospitalizados en unidades de pediatría del Hospital Virgen Macarena. Index de Enfermería, 18(4), 243 - 245.

Violant, V., Molina, M. y Pastor, C. (2009). Pedagogía Hospitalaria: Necesidades, ámbitos y metodología de intervención. Santiagode Chile: Ministerio de Educación, Gobierno de Chile. 